This article is dedicated to the memory of Vyacheslav Borisovich Priezzhev

\title{
Kinetic Processes in Fullerene Solutions
}

\author{
T. V. Tropin ${ }^{a, *}$, V. L. Aksenov ${ }^{a}$, and J. W. P. Schmelzer ${ }^{b}$ \\ ${ }^{a}$ Frank Laboratory of Neutron Physics, Joint Institute for Nuclear Research, Dubna, 141980 Russia \\ ${ }^{b}$ Institute of Physics, University of Rostock, Rostock, 18051 Germany \\ *e-mail: ttv@jinr.ru
}

Received March 23, 2020; revised June 19, 2020; accepted June 19, 2020

\begin{abstract}
In solutions, fullerenes, carbon nanoparticles with size of the order of one nanometer, exhibit a number of interesting properties and kinetic effects. A large part of these effects is connected with the ability of these macromolecules to form aggregates (or clusters), in which the nanoparticles are bound together by dispersion interactions. In this review, we present results of modeling of the kinetics of clusters formation and growth in fullerene $\mathrm{C}_{60}$ solutions of different polarity. The basic approach is the numerical solution of a system of kinetic equations of nucleation theory, applied here for the description of aggregation of fullerenes and accompanying effects. The non-monotonous time dependence of fullerene concentration during dissolution was investigated. A molecular-colloidal solution transition in polar systems is described. In addition, a model description of the critical effect of cluster decomposition after water addition to certain fullerene solutions is presented.
\end{abstract}

DOI: $10.1134 / \mathrm{S} 1063779621020076$

\section{INTRODUCTION}

The processes of aggregation (coagulation, clusterization) of particles of different sizes are known and were investigated for a very long time. In 20th century considerable results in understanding, description and application of these phenomena were attained. One of the striking examples of this type of behavior are the solutions of fullerenes in different liquids. Fullerenes, a novel allotropic form of carbon, were discovered at the end of 20th century [1]. They represent a class of symmetric molecules with sizes of around one nanometer. The most stable and abundant among them is the $\mathrm{C}_{60}$ molecule (Fig. 1), formed by 60 carbon atoms, located at the vertices of a highly-symmetric figurethe truncated icosahedron ("soccer ball" with the radius $\sim 0.35 \mathrm{~nm}$ ) [2]. Unlike the "classic" allotropic forms of carbon (graphite, diamond) fullerenes dissolve in a variety of different liquids [3]. This property of fullerenes gave rise to additional interest, connected initially with their obtaining and purifications, and further with application of the antioxidant and anticancer properties of these macromolecules.

At the same time, in solutions fullerenes exhibit several additional peculiar properties, including formation of stable or dynamically changing clusters of $\mathrm{C}_{60}$ molecules [4]. During last 30 years a considerable amount of experimental data on these phenomena was accumulated, the structure of the clusters and the kinetics of their growth were investigated [5-7]. In this analysis, not only confirming but partly also contradicting each other results are reported. These contradictions in results is an evidence of the complicated character of dissolution and cluster growth in these solutions, and on the considerable influence of external factors on these processes. By mentioned reasons, the development of theoretical description of kinetic processes, occurring in fullerene solutions, first of all, the formation and growth of clusters, is indeed an interesting and quite actual problem.

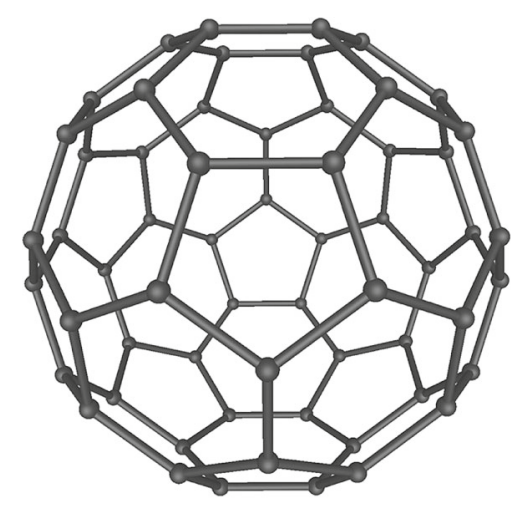

Fig. 1. The structure of $\mathrm{C}_{60}$ fullerene molecule. 
In the present work, we discuss the methods for description of kinetics of cluster formation and growth in different solutions of fullerenes. These methods, based on the theory of nucleation, were developed in scope of joint research of scientists from JINR (FLNP and BLTP), Taras Shevchenko National University of Kyiv (Ukraine) and University of Rostock (Germany). For a considerable period, V.B. Priezzhev was actively participating in these investigations.

Structure of the review is the following: because the basis of the presented investigations are the modified kinetic equations for the cluster-size distribution function of the classical theory of nucleation and cluster growth, in Section 2 their derivation, main properties and results are presented. It is shown further how this general method can be applied to processes of segregation in fullerene solutions. In Section 3, the results of theoretical description of kinetic processes in certain fullerene solutions are presented. At the beginning of each subsection a brief information on the systems considered is given-experimental observations that are described within the theoretical models. At the end of the article, the perspectives of the conducted research are discussed.

\section{THEORY OF NUCLEATION AND GROWTH}

In this section, we review the main concepts of the theory of cluster formation and growth in different media. As a first step in this direction, the basic ideas underlying the theory of nucleation are summarized. As essential ingredients, the work of cluster formation, kinetic equations of nucleation theory, probabilities of particle attachment/detachment are discussed. The first three subsections constitute, in fact, the classical theory of nucleation. In the fourth subsection, we review the Zeldovich-Frenkel equation. The Section is concluded by discussion of the different stages in the evolution of a system undergoing a first-order phase transition of the considered type, specifically the stages of growth that follow nucleation.

\subsection{The Work of Cluster Formation}

The discovery and thorough investigation of supercooled liquids and supersaturated vapors were performed already in 19th century (see e.g. Volmer [8]). Relying on these discoveries Gibbs was able to formulate the thermodynamic basis of the theory of nucleation. After 150 years the theory has considerably developed, and includes now the kinetic description of not only the processes of formation, but also of growth of particles of the new phase. Here we describe the basis of this theory in its applications to description of kinetics of formation and growth of aggregates composed of atoms, molecules, colloids in solutions. This basis was laid, following the analysis of Gibbs [9, 10], in works of Volmer and Weber [11], Farkas [12], Kai- schew and Stranski [13, 14], Becker and Döring [15], and later by Zeldovich [16], Frenkel [17], Turnbull and Fisher [18].

Nucleation (formation of the nuclei of new phase) the first stage of a phase transition-in many respects defines the character and properties of this phenomenon. Thus, its precise description is of considerable interest [19]. The formation of nuclei in homogeneous phases is a process of fluctuational character. One of Gibbs basic hypotheses is that these nuclei, as small as they can be, are equilibrium particles of the newly evolving phase, inside the homogeneous metastable medium - the initial phase. If we are considering the conditions sufficient for the phase transition (supercooled liquid, supersaturated vapor or solution), then thermodynamically stable (and thus favorable) state is the new phase. It follows that for atoms or molecules in the system the following inequality between the chemical potentials holds: $\mu_{\beta}(p, T, X)>\mu_{\alpha}(p, T, X)$. Here subscript $\beta$ identifies the properties of the initial, and subscript $\alpha$ of the newly forming phase, $p$ is the pressure, $T$ is temperature and $X$ is a set of additional thermodynamic parameters, that define the systems state. The difference $\Delta \mu=\mu_{\beta}(p, T, X)-\mu_{\alpha}(p, T, X)$, sometimes denoted as the driving force of the transition, in many respects defines its rate.

When pressure, temperature and particles number are fixed for a given solution, its state is described by the Gibbs free energy, $G(p, T)$. The change of $G$ on formation of a single nucleus (cluster) of a new phase from $n$ particles of the initial phase (so-called work of formation) is [20]:

$$
\Delta G(n)=G_{\text {nucl }}(n)-n \mu_{\beta}(p, T, X),
$$

where $G_{\text {nucl }}(n)$ is the contribution of the cluster consisting of $n$ particles to Gibbs free energy of the whole system. The quantity $\Delta G(n)$, in its general form (2.1), defines the minimum work for formation of this nucleus in a reversible change of systems state. Nuclei formation in the initial phase is a fluctuational process-small clusters (usually, nanoparticles) with a density close to the new phase's density appear stochastically in random places in the matrix. The work of their formation, $\Delta G(n)$, defines the equilibrium cluster size distribution in the system [21]:

$$
f^{\mathrm{eq}}(n) \sim \exp \left(-\frac{\Delta G(n)}{k_{\mathrm{B}} T}\right),
$$

where $f(n)$ here and further is the cluster size distribution function ( $n$ is the number of particles in the cluster). It is equal to the number of clusters, consisting of $n$ monomers (atoms, molecules, particle of the new phase), in the unit volume.

The widely used approximation for the work of cluster formation is the so-called liquid drop model $[8,22]$ :

$$
\Delta G(n)=-n \Delta \mu+\sigma A,
$$


where $A$ is the surface area of the cluster, and $\sigma$ is the surface tension at the planar interface between two phases. The $\Delta G(n)$ dependence, described by (2.3), is presented on Fig. 2. Because the phase transition is thermodynamically favorable $(\Delta \mu>0)$, sufficiently large particles of new phase will have free energies lower than the corresponding number of particles of the initial phase. However, for the small nuclei a big role plays the phase boundary. Indeed, the number of particles at the interface in this case is a considerable part of the whole number of particles in nucleus. The particles at the phase boundary are in energetically less favorable state, than the particles in the bulk of new phase. As a result, a positive work, spent for the formation of phase boundary, is required for the formation of small clusters of the new phase.

One can rewrite (2.3) by providing an expression for surface area $A$ [23]:

$$
\begin{gathered}
\Delta G(n)=-n \Delta \mu+4 \pi \sigma\left(\frac{3}{4 \pi} \omega_{\alpha}\right)^{2 / 3} n^{2 / 3} \\
=-n \Delta \mu+\alpha_{2} n^{2 / 3}, \quad \alpha_{2}=4 \pi \sigma\left(\frac{3}{4 \pi} \omega_{\alpha}\right)^{2 / 3},
\end{gathered}
$$

where $\omega_{\alpha}$ is the volume, corresponding to a single monomer in the new phase. We have also introduced a new designation $\alpha_{2}$. In metastable state the first term in (2.4) is always negative, the second-always positive, thus it follows that a maximum (the so-called nucleation barrier) is always present in the $\Delta G(n)$ dependence (see Fig. 2). This barrier defines the critical cluster size $n_{\mathrm{c}}$ and the work of critical cluster formation $\Delta G_{\mathrm{c}}$ :

$$
n_{\mathrm{c}}=\left(\frac{2 \alpha_{2}}{3 \Delta \mu}\right)^{3}, \Delta G_{\mathrm{c}}=\Delta G\left(n_{\mathrm{c}}\right)=\frac{1}{3} \alpha_{2} n_{\mathrm{c}}^{2 / 3} .
$$

The clusters, forming in the system due to fluctuations, can be divided into three categories: subcritical $\left(n<n_{\mathrm{c}}\right)$, critical clusters $\left(n=n_{\mathrm{c}}\right)$, and supercritical $\left(n>n_{\mathrm{c}}\right)$ nuclei of the new phase. The systems state is unsaturated for the subcritical clusters, leading to their decomposition, while the supercritical clusters in general grow in size. The critical cluster $\left(n=n_{\mathrm{c}}\right)$ is in unstable thermodynamic equilibrium with the surrounding phase. It follows that the probability of stochastic formation of the critical cluster in the system is:

$$
w \sim \exp \left(-\frac{\Delta G_{\mathrm{c}}}{k_{\mathrm{B}} T}\right)
$$

and, thus, the nucleation rate-number of formed nuclei of new phase per time unit is given by a relation of the form:

$$
J=J_{0} \exp \left(-\frac{\Delta G_{\mathrm{c}}}{k_{\mathrm{B}} T}\right)
$$

Thus, expression (2.7) provides possibility for investigations of nucleation rate $J$ dependence on ther-

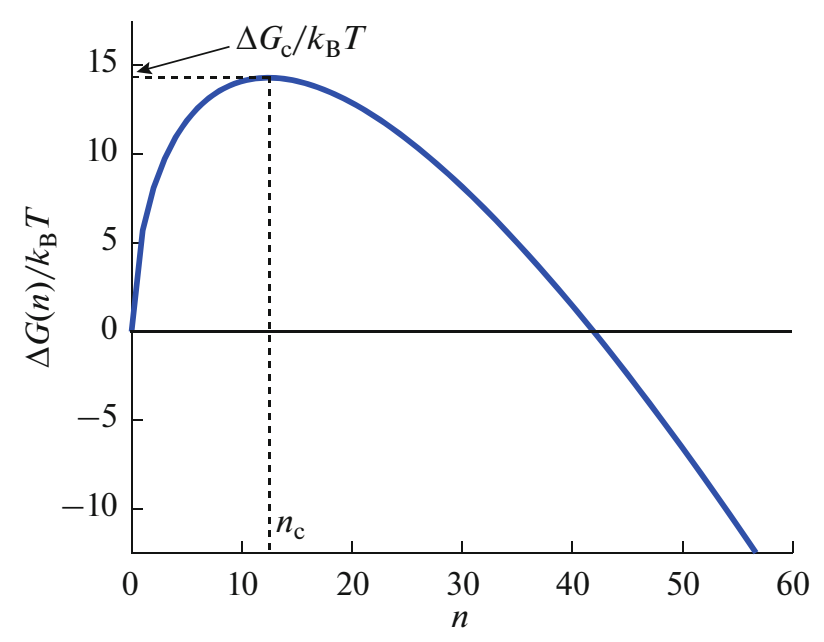

Fig. 2. Dependence of the work of formation of a nucleus (cluster) in the initial phase on the number of particles $n$.

modynamic properties of the system. It was first formulated by Volmer and Weber [11]. Further development of nucleation theory is connected with the composition of the system of kinetic equations, description of growth and decay of the new phase nuclei, and the consequent specification of the preexponential term $J_{0}$.

\subsection{Kinetic Approach}

For the first time in works by Farkas [12], Kaischew and Stranski [13, 14], Becker and Döring [15] the character of formation of nuclei is described in frame of chain collisions and merges of separate atoms or molecules into clusters consisting-in the simplest case of phase formation in one-component systemsof $n$ ambient phase units [12,15]. This approach can be employed also for multi-component systems [24], however, here we will restrict the considerations to the one-component case. Let us define the cluster size distribution function, $f(n, t)$, and write down the kinetic equation, supposing that the decomposition and growth of nuclei is possible only as the result of their binary reactions with monomers-separate particles of the ambient phase:

$$
\begin{gathered}
\frac{\partial f(n, t)}{\partial t}=w_{n-1, n}^{(+)} f(n-1, t)+w_{n+1, n}^{(-)} \\
\times f(n+1, t)-w_{n, n+1}^{(+)} f(n, t)-w_{n, n-1}^{(-)} f(n, t),
\end{gathered}
$$

where $w_{n, n+1}^{(+)}$is the probability for the cluster of size $n$ to attach a monomer per time unit, and $w_{n, n-1}^{(-)}-$on the opposite, is the probability, that a monomer is detached from the cluster of size $n$. Introducing $J(n)$, the fluxes in cluster size space:

$$
J(n, t)=w_{n, n+1}^{(+)} f(n, t)-w_{n+1, n}^{(-)} f(n+1, t),
$$


we can rewrite Eq. (2.8) in the form:

$$
\frac{\partial f(n, t)}{\partial t}=-\{J(n, t)-J(n-1, t)\}, \quad n \geq 2 .
$$

Becker and Döring [15], by considering the stationary state of the system $(\forall n>1: J(n-1)=J(n))$, and using the detailed balance approximation, employed for the specification of the coefficients of emission (see the subsequent subsection), obtained the following expression for the nucleation rate with a defined pre-exponential term:

$$
I(t)=\frac{a_{0} f(1, t)}{n_{\mathrm{c}}} \sqrt{\frac{1}{3 \pi} \frac{\Delta G_{\mathrm{c}}}{k_{\mathrm{B}} T}} \exp \left(-\frac{\Delta G_{\mathrm{c}}}{k_{\mathrm{B}} T}\right),
$$

where $a_{0}$ is the probability of particle attachment to the cluster (per time unit and unit area). It should be noted, that Eq. (2.7) was obtained from general considerations by Volmer and Weber, who have obtained $I_{0} \sim w^{(+)} f(1)$ as the expression for the pre-exponential term.

\subsection{Ratio of Probabilities of Clusters Growth and Decay}

One of the most discussed questions of theory of nucleation and growth is the method of derivation of the $w_{n, n+1}^{(+)}, w_{n, n-1}^{(-)}$Eq. (2.8) coefficients. Indeed, defining the expressions for probabilities of changes of cluster sizes completes the system of kinetic equations, and thus determines the success of the theory. While certain expressions for $w_{n, n+1}^{(+)}$can be relatively easy derived from the physical consideration of the models of the system (bubble growth in liquid, a drop in vapor, crystal nucleus in solution), a similar approach for determining $w_{n, n-1}^{(-)}$does not bear good results. Therefore, starting from the earliest works, basing on the detailed balance approximation, the following approach was proposed. From the equality of the corresponding transitions, we can write a chain of expressions of the form:

$$
w_{n+1, n}^{(+)} f^{\mathrm{eq}}(n)=w_{n+1, n}^{(-)} f^{\mathrm{eq}}(n+1)
$$

or, using (2.2),

$$
\frac{w_{n+1, n}^{(+)}}{w_{n+1, n}^{(-)}}=\exp \left(-\frac{\Delta G(n+1)-\Delta G(n)}{k_{\mathrm{B}} T}\right),
$$

where the pre-exponential factor was canceled by supposing that its dependence on $n$ and system properties is much weaker, than of the exponential term. Equation (2.13) allows obtaining coefficients $w_{n, n-1}^{(-)}$by using the expressions for work of cluster formation and probability of cluster growth. Let us note that this method of derivation is applicable only for the stationary, moreover, equilibrium states, thus its value is strongly decreased. Nevertheless, it is widely used for calculations and kinetic equations, that is, the conclusions justified for equilibrium systems, were quite bravely extrapolated to non-equilibrium states without sufficient theoretical foundation. It should be noted, that some time ago an alternative approach was proposed [20, 25-27], allowing to obtain the ratio of growth and decay probabilities in a generally nonequilibrium case. Using the concept of virtual states (of the ambient phase), that are in equilibrium with respect to the different sized clusters of novel phase, it was successfully shown that the expression (2.13) holds in fact for the general case of systems evolution. In this way, one serious problem in the development of the fundamental relations in the description of the kinetics of phase formation processes could be resolved (cf. e.g. [28]).

Having at one's disposal the relations between the coefficients of aggregation and dissolution, (2.13), the analysis (analytical or numerical) of the system of kinetic Eqs. (2.8) can be started after the expression for monomer attachment, $w_{n, n+1}^{(+)}$, is defined. For example, for the classical case of vapor condensation, the following expression holds:

$$
w_{n, n+1}^{(+)}=\frac{1}{4} N_{1}\left(\frac{8 k_{\mathrm{B}} T}{\pi m}\right)^{1 / 2} 4 \pi R_{n}^{2},
$$

where $N_{1}$ is the volume density of separate vapor particles (that is $\left.N_{1}=f(1, t)\right), m$ is their mass, $R_{n}$ is drop radius. In the present work a different expression for $w_{n, n+1}^{(+)}$will be used (see below). It defines the probability of molecules attachment to the growing solid cluster in fullerene solution.

\subsection{Zeldovich-Frenkel Equation}

The mathematical analysis of Eqs. (2.8), (2.10), especially the kinetic effects, connected, for example, with the change of systems state (external parameters, composition, supersaturation) or the dependence of nucleation on initial and boundary conditions, is difficult because of the discrete character of these equations. Therefore, following the work by Zeldovich [16], a vast research by employing the continuity approximation was made. Considering $n$ in (2.8) as a continuous variable, one can expand the last two terms. Leaving first and second order terms in the series, we obtain a Fokker-Planck type equation for $f(n, t)[16,17,29]$ :

$$
\begin{gathered}
\frac{\partial f(n, t)}{\partial t}=-\frac{\partial}{\partial n}\left\{\left(w_{n-1, n}^{(+)}-w_{n+1, n}^{(-)}\right) f(n, t)\right\} \\
+\frac{1}{2} \frac{\partial^{2}}{\partial n^{2}}\left\{\left(w_{n-1, n}^{(+)}+w_{n+1, n}^{(-)}\right) f(n, t)\right\} .
\end{gathered}
$$

Therefore, in the dimension of cluster sizes, the quantity $v_{n}=\left\langle w_{n-1, n}^{(+)}-w_{n+1, n}^{(-)}\right\rangle$corresponds to the velocity, e.g. mean rate of growth of clusters of size $n$, 
and the quantity $D_{n}=\left(w_{n-1, n}^{(+)}+w_{n+1, n}^{(-)}\right) / 2$ is the diffusion rate (stochastic movements in cluster size space). The diffusional part of Eq. (2.15) is significant near the critical cluster size. Indeed, we can write by definition:

$$
\left.\left.w_{n-1, n}^{(+)}\right|_{n \simeq n_{\mathrm{c}}} \cong w_{n+1, n}^{(-)}\right|_{n \simeq n_{\mathrm{c}}}
$$

and, therefore, $v_{\mathrm{n}} \approx 0$, while $D_{n}=\left.w_{n-1, n}^{(+)}\right|_{n=n_{\mathrm{c}}}$. Far from $n_{\mathrm{c}}$, specifically when $n>n_{\mathrm{c}}$, the significance of stochastic term is lost and cluster growth is defined by the value of $v_{\mathrm{n}}$. We can write then:

$$
\left.\frac{\partial f(n, t)}{\partial t}\right|_{n>n_{\mathrm{c}}} \cong-\frac{\partial}{\partial n}\left\{\left(w_{n-1, n}^{(+)}-w_{n+1, n}^{(-)}\right) f(n, t)\right\},
$$

i.e. the character of evolution of the cluster size distribution is defined by the continuity equation in clustersize space.

Neglecting the second term in expansion and using (2.9), (2.11) and (2.12), the widely known ZeldovichFrenkel equation is obtained [16, 17]:

$$
\frac{\partial f(n, t)}{\partial t}=\frac{\partial}{\partial n}\left\{w_{n, n+1}^{(+)} f^{\mathrm{eq}}(n) \frac{\partial}{\partial n}\left(\frac{f(n, t)}{f^{\mathrm{eq}}(n)}\right)\right\}
$$

or, in another form:

$$
\frac{\partial f(n, t)}{\partial t}=\frac{\partial}{\partial n}\left\{w_{n, n+1}^{(+)}\left[\frac{\partial f(n, t)}{\partial n}+\frac{f(n, t)}{k_{\mathrm{B}} T} \frac{\partial \Delta G(n)}{\partial n}\right]\right\} .
$$

Equation (2.19) has the form of diffusion equation in the force field, where the derivative $\partial \Delta G(n) / \partial n$ describes the generalized thermodynamic "force". The Zeldovich-Frenkel equation, widely equivalent in its consequences to the kinetic Eqs. (2.8), allows obtaining important analytical estimates, not easily attainable from analysis of the discrete equations. For example, some particular cases of variable supersaturation were considered, allowing, for example, estimating the induction time of nucleation.

\subsection{Cluster Formation and Growth in Solutions}

Here we consider the application of nucleation theory for describing the formation and growth of clusters in colloidal or nanoparticle solutions. To do this, we must provide expressions for the driving force $\Delta \mu$ and the probability of monomer attachment to the cluster.

Considering a perfect solution, we can write [16]:

$$
\Delta \mu=k_{\mathrm{B}} T \ln \left(\frac{c_{\mathrm{mon}}(t)}{c_{\mathrm{eq}}}\right),
$$

where $c_{\text {mon }}(t) \equiv f(1, t)$, and $c_{\text {eq }}$ is the equilibrium concentration of dissolved particles in the solvent at the planar interface with solid phase. The expression for $w_{n, n+1}^{(+)}$is classically obtained by considering the flux of particles in the solution to the cluster with radius $R_{n}$, positioned at the origin $[16,19,23,29]$. The expres-

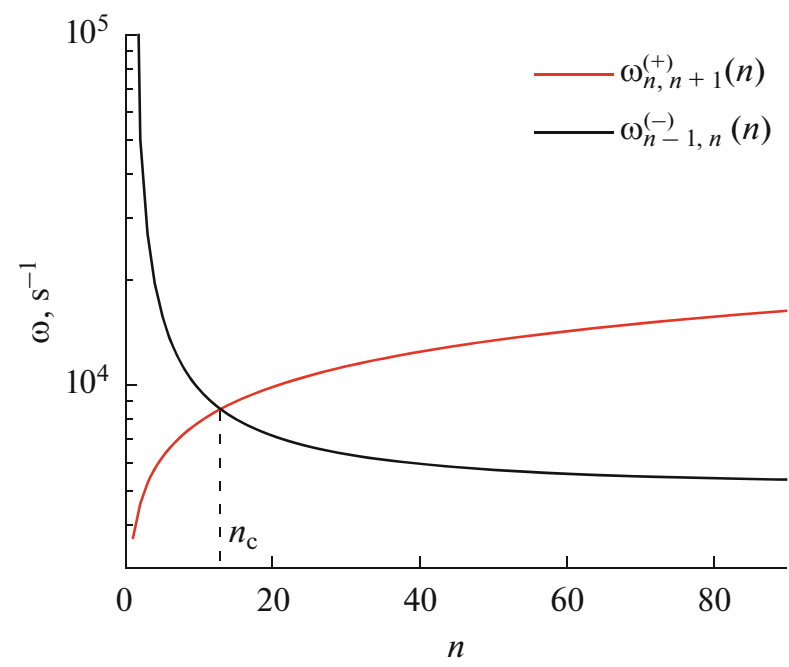

Fig. 3. Dependence of the probabilities of growth (red curve) and decay (black curve) on the cluster size for the case of clusters formation and growth in solution.

sions for $w_{n-1, n}^{(+)}$for different regimes of cluster growth were obtained. In case of diffusion-limited aggregation regime, we have:

$$
w_{n-1, n}^{(+)}=4 \pi D c_{\text {mon }}(t) r n^{1 / 3},
$$

where $r$ is the size of a single segregating particle, $D$ is its diffusion rate in the solvent. For nucleation and growth limited by the kinetics of particle attachment to the cluster, we can write:

$$
w_{n, n+1}^{(+)}=4 \pi D^{*} c_{\text {mon }}(t) r\left(\frac{\omega_{\mathrm{s}}}{\omega_{\mathrm{m}}}\right) n^{2 / 3},
$$

where $D^{*}$ is the effective diffusion rate that defines the speed of particle attachment to the cluster (commonly, the relation $D^{*}<D$ holds), $\omega_{\mathrm{s}}, \omega_{\mathrm{m}}$ are volumes, corresponding to the particle in solution and in the cluster.

The calculated curves for the model solution of fullerenes are presented on Fig. 3. It is clearly seen that for $n<n_{\mathrm{c}}$ decomposition of a cluster is more probable than its growth, and for $n>n_{\mathrm{c}}$ the opposite statement is true. It should be noted, that the prefactors of $n$ in Eqs. (2.21), (2.22) are used for introducing a dimensionless time scale for the kinetic equations. Further, in presenting the results of numerical calculations, the dimensionless time scale will still be denoted as $t$ (unless it is specifically stated).

The dependencies, presented on Figs. 2 and 3, evolve with time in the real systems, specifically in weak solutions. Indeed, the monomer concentration $c_{\text {mon }}(t)$ continuously decreases due to formation and growth of supercritical clusters. This changes the driving force in the system (see (2.20)), and also $\Delta G(n)$, $w_{n, n+1}^{(+)}$and $w_{n+1, n}^{(-)}$dependencies. While the $c_{\text {mon }}(t)$ 


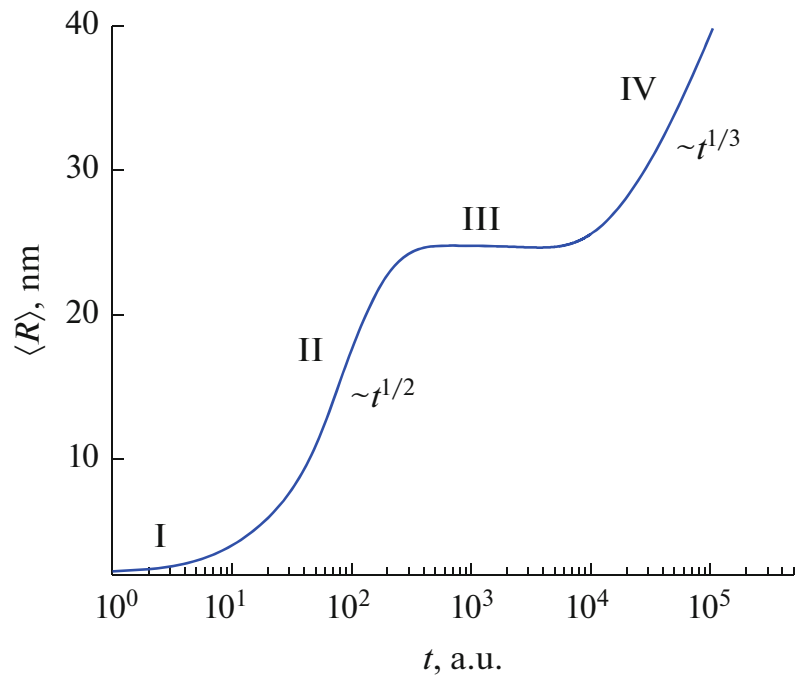

Fig. 4. Time dependence of the mean cluster radius in the system during nucleation and growth stages [30]. Dependencies $\sim t^{1 / 2}$ and $\sim t^{1 / 3}$ correspond to the diffusion-limited aggregation regime, the first one to independent growth of the clusters, the second on to competitive growth or coarsening.

dependence does not play a major role at early stages of systems evolution-during nucleation, for the stages of growth of large clusters, the decrease of supersaturation leads to new kinetic effects. The system passes through several stages of evolution, which can be distinguished by investigating the dynamics of several characteristics of the cluster state, for example, of the mean cluster size in solution, $\langle R\rangle$. Its value is defined by the expression:

$$
\langle R(t)\rangle=\frac{1}{\sum_{n=1}^{\infty} n f(n, t)} \sum_{n=1}^{\infty} R_{n} f(n, t) .
$$

Two comments regarding Eq. (2.23) must be made. First, while this expression defines the "true" mean radius of the particles in the system, one should take into account, that the observed (measured) average sizes are influenced by the resolution limit of the experimental method applied. Generally, the resolution function limits the values of measured properties both from above and below. Indeed, in (2.23) the sum is already limited by the size of the largest clusters present in solution, yet this size can exceed the resolution limit of the experimental method. Second, a large part of summation in Eq. (2.23) consists of $f(n, t)$ with small $n$ values, which correspond to monomers and stochastically formed small clusters $\left(n<n_{\mathrm{c}}\right)$. These small aggregates do not characterize the evolution of the system, but may strongly influence the value of $\langle R\rangle$. The evolution of the system after the nucleation stage is well represented if the modified expression for the average cluster size is used:

$$
\langle R(t)\rangle=\frac{1}{\sum_{n=n_{\min }}^{n_{\max }} n f(n, t)} \sum_{n=n_{\min }}^{n_{\max }} R_{n} f(n, t),
$$

where the summation limits $n_{\min }$ and $n_{\max }$ are defined by the described above considerations (e.g. experimental method limits).

Numerical solution of Eqs. (2.8) is a powerful tool that allows to relatively easy follow the systems transition from nucleation to the next stages of cluster growth. To proceed further the system of equations must be supplemented by the initial and boundary conditions. Often, these are taken in the form:

$$
\left\{\begin{array}{l}
f(1,0)=c_{0}, \\
f(n, 0)=0, \quad n>1, \quad \sum_{n=1}^{\infty} f(n, t) n=c_{0} .
\end{array}\right.
$$

The initial conditions then correspond to state of the system without clusters-the whole dispersing phase is in the form of separated particles, monomers. The boundary conditions in (2.25) correspond to a constant in time concentration of solution.

The results of the model calculations for the supersaturated solution are presented on Fig. 4. Systems evolution proceeds, generally, through four stages. The first stage consists of the so-called nucleation time-lag (or induction time) and the steady-state nucleation. First, the exponential incipient distribution function for nucleation is formed out of the initial distribution (2.25). Further, steady-state nucleation proceeds, and a sufficient concentration of supercritical clusters is formed. These clusters will growth further. During stage II, the independent growth stage, supercritical clusters grow, consuming the supersaturation $c(t) / c_{\mathrm{eq}}$. Thus, critical cluster size $n_{\mathrm{c}}$ "pursues" the cluster population. Finally, it "reaches" and surpasses some of the growing clusters, making them subcritical. At stage III the system is preparing for a new type of growth (and the cluster size distribution function). Larger clusters in the system will further grow by consuming the monomers detached from smaller clusters. During stage III, the average cluster size in solution stays constant. Further, the final stage of system evolution, the Ostwald ripening stage [31], begins. During this stage, the competitive growth of aggregates continues, but the mean cluster size is now increasing (following a power-law dependence). The final state of the system is a single huge "cluster" of the novel phase (in equilibrium with $c_{\text {eq }}$ monomers). This final stage is characteristic for several first-order phase transitions. Analytical investigation of kinetics of clusters growth at this stage was performed by V.V. Slezov and I.M. Lifshits [32, 33]. They have obtained, that for the diffusion-limited aggregation, the so-called " $t$ t/3 law" holds during stage IV:

$$
\langle R\rangle^{3}=\left\langle R_{0}\right\rangle^{3}+\frac{4}{9} D \alpha t,\langle R\rangle \gg\left\langle R_{0}\right\rangle,
$$


where $\left\langle R_{0}\right\rangle$ is the average cluster radius at the end of the stage of independent growth. The analytical expressions for the cluster size distribution functions were also obtained:

$$
f(R, t)=N(t) P\left(\frac{R}{\langle R\rangle}\right) \frac{1}{\langle R\rangle}, \quad P(u)= \begin{cases}\frac{3^{4} e}{2^{5 / 3}} \frac{u^{2} \exp \left(-\frac{1}{1-(2 / 3) u}\right)}{(u+3)^{7 / 3}((3 / 2)-u)^{11 / 3}} & 0<u<3 / 2, \\ 0, & u \geq 3 / 2\end{cases}
$$

where $N(t)$ is the time dependence of the number of particles in the system.

The obtained kinetic equations and analytical estimates allow performing a qualitative and quantitative description of kinetics of first-order phase transition, and, as a special case, the kinetics of particles aggregation in solutions. Application of this approach to description of fullerenes $\mathrm{C}_{60}$ aggregation in different solutions, and of some complementary phenomena, was made in collaborative research by the joint group of scientists from Russia (FLNP \& BLTP, JINR), Germany and Ukraine during last 15 years. In the next Section, we present a review of several results obtained.

\section{KINETICS OF CLUSTER GROWTH IN FULLERENE SOLUTION}

In low-polar and polar liquids, fullerenes, unlike other allotropic forms of carbon, develop a relatively good solubility [34, 35]. At first there was an inconsistency within various experimental data for $\mathrm{C}_{60}$ and $\mathrm{C}_{70}$ solutions [4, 36, 37], and a considerable difference in first measurements of the solutions macroscopic parameters (diffusion coefficients, solubility) $[3,34,36]$. It is known presently, that one of the reasons of the inconsistencies is the cluster formation and growth in these systems [4, 38, 39]. In particular, in low-polar liquids $(\varepsilon<20)$, cluster growth strongly depends on dissolution conditions, especially on external influence (ultrasound, oxygenation and light). Thus, a consequent description of cluster growth in these systems is an actual and interesting problem.

For the first time, nucleation theory was applied in $[30,40]$ for description of cluster state of fullerenes in solution, the liquid drop and limited growth models were considered [10-13]. The following investigations allowed describing several of the observed effects $[7,41,42]$. In this Section, we review three examples of applications of kinetic theory of clusters formation and growth to fullerene solutions.

\subsection{Non-Monotonous Fullerenes Concentration Dependence during Dissolution in Low-Polar Liquids}

One of the most interesting effects, connected with non-equilibrium cluster formation in low-polar solutions of fullerenes, is the non-monotonous character of time dependence of fullerene concentration during dissolution. In the saturated solution of $\mathrm{C}_{60}$ in carbon disulfide $\left(\mathrm{CS}_{2}\right)$ after the maximum concentration is reached within first three days, fullerene concentration decreases (at a rate of $\sim 20 \%$ per day) towards the stable equilibrium value. This effect was observed by spectrophotometry of visual and ultraviolet light [43] (UV-Vis) and later confirmed during small-angle neutron scattering experiments [44-46]. In [47] a model of fullerene $\mathrm{C}_{60}$ dissolution, capable of describing this effect, was developed. Within this model, fullerene dissolution is described together with accounting of sedimentation of large particles and cluster growth in solution. Numerical solution of kinetic equations allows making a detailed analysis of dissolution regimes. Specifically, one of these regimes qualitatively describes the non-monotonous dependence of fullerene concentration in carbon disulfide.

To apply the equations for describing the kinetics of fullerene dissolution in this case the particle flows from and to the solid phase must be modeled. First, we introduce a flow of fullerene molecules from the solid phase to solution. It stops when the saturation concentration is reached. This leads to the following change of conditions (2.25):

$$
\begin{gathered}
f(n, 0)=0, \forall n ; \sum_{n=1}^{\infty} n f(n, t)=c(t) ; \\
\dot{f}(1, t)= \begin{cases}\dot{c}, & c(t)<c^{\prime} \\
0, & c(t)>c^{\prime}\end{cases}
\end{gathered}
$$

where $c^{\prime}$ is saturation concentration; $\dot{f}(1, t)$ is rate of $\mathrm{C}_{60}$ particles flow from the solid into the solution at time $t$. As a first approximation, we neglect the possible $\dot{c}(t)$ dependence: let fullerene concentration in solution grow linearly with time, $\dot{c}=$ const. Also, in Eqs. (2.8) we must account for sedimentation of huge clusters. Let $n^{\prime}$ be the minimum size of clusters subject to sedimentation in solution. This process will be accounted by the term $-\left(e^{n / n^{\prime}}-1\right) f\left(n, t^{\prime}\right)$ in $(2.8)$, for each $n>n^{\prime}$. Indeed, in this case the sedimentation rate grows exponentially with cluster size. 

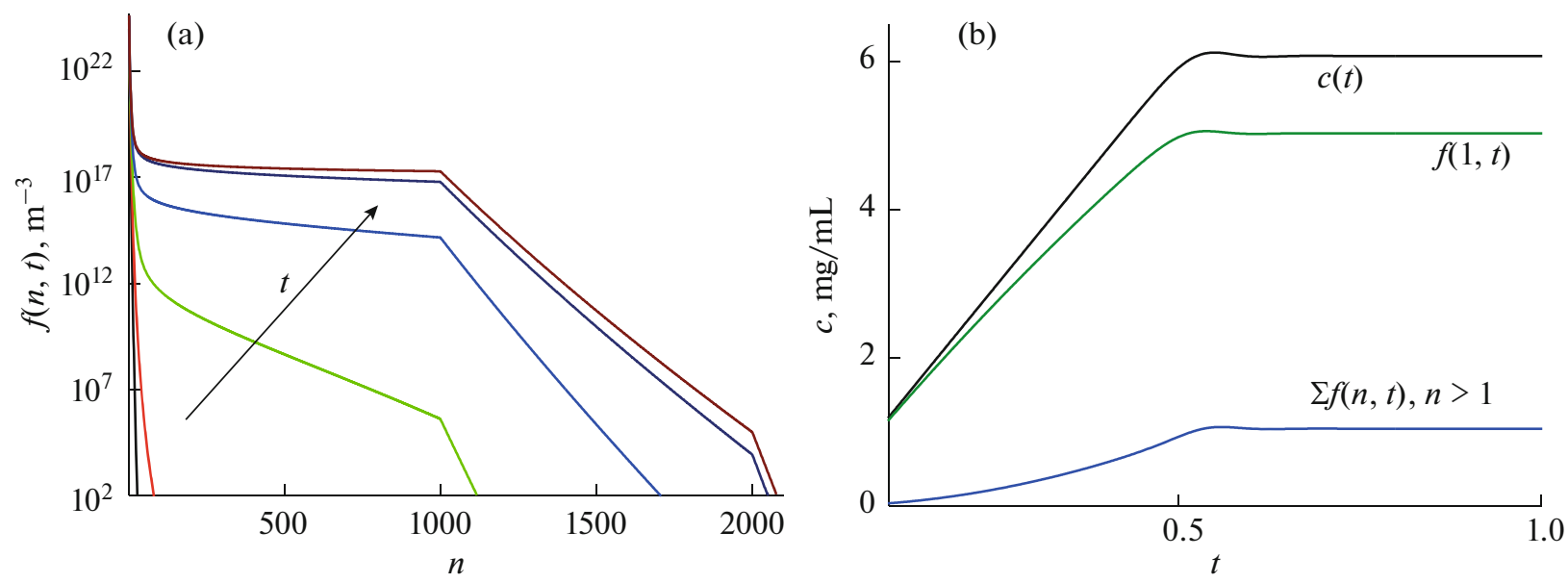

Fig. 5. (a) Time evolution of the $\mathrm{C}_{60}$ cluster size distribution. (b) Time dependencies of $c(t)$-total fullerene concentration, $f(1, t)$-monomer $\mathrm{C}_{60}$ concentration and $\Sigma f(n, t), n>1$-fullerene clusters concentration in solution. Model parameters: $c^{\prime}=5.7 \times 10^{24} \mathrm{~m}^{-3}, c_{\mathrm{eq}}^{(\infty)}=5.7 \times 10^{23} \mathrm{~m}^{-3}, n^{\prime}=1000, \dot{c}=10^{22} \mathrm{~m}^{-3} / d t^{\prime}$.

As a result, we obtain the following equations:

$$
\left\{\begin{array}{l}
\frac{\partial f(n, t)}{\partial t}=w_{n-1, n}^{(+)} f(n-1, t)+w_{n+1, n}^{(-)} f(n+1, t) \\
-\left(w_{n, n+1}^{(+)}+w_{n, n-1}^{(-)}\right) f(n, t), \quad n \leq n^{\prime}, \\
\frac{\partial f(n, t)}{\partial t}=w_{n-1, n}^{(+)} f(n-1, t)+w_{n+1, n}^{(-)} f(n+1, t) \\
-\left(w_{n, n+1}^{(+)}+w_{n, n-1}^{(-)}+\left(e^{n / n^{\prime}}-1\right)\right) f(n, t), n>n^{\prime} .
\end{array}\right.
$$

In [47] the system of Eqs. (3.2) was solved numerically with the additional conditions (3.1) for different sets of parameters $c^{\prime}, c_{\mathrm{eq}}^{(\infty)}, \dot{c}, n^{\prime}$. The evolution of the fullerene concentration in solution $c(t)$ was obtained from the cluster size distribution function $f(n, t)$. Four regimes of fullerenes dissolution were discovered, two of which are characterized by a monotonous growth of $c(t)$, and other two produce non-monotonous dependencies (for more details, see [47]). One of these four regimes corresponds to the experimental observations (Fig. 5).

The proposed model describes the kinetics of dispersed systems in a general form. Its special case are the considered here fullerene solutions in low-polar solvents.

\subsection{Influence of Fullerene-Solvent Complexes Formation on Kinetics of Cluster Growth}

In some polar fullerene solutions, a kinetic transition from molecular to the colloidal state is observed. Good examples of this type of behavior are $\mathrm{C}_{60}$ solutions in nitrogen-containing solvents $\mathrm{N}$-methylpyrrolidone $\left(\mathrm{C}_{5} \mathrm{H}_{9} \mathrm{NO}, \mathrm{NMP}\right)[48,49]$ and pyridine $\left(\mathrm{C}_{5} \mathrm{H}_{5} \mathrm{~N}\right)$. The initial state (a molecular solution) is unstable. Within a certain period (minutes, sometimes hours) a slow transition to a stable colloidal solution of fullerenes occurs. Experimental investigations of these systems $[48,50]$ showed that the formation of chargetransfer complexes between fullerene and solvent molecules takes place $[38,48,51]$. In $[42,52,53]$ a model of aggregation kinetics in these solutions was developed. Modification of the basic equations to account for the influence of complexes on clusters formation and growth is made by introducing an additional model parameter-the time of complex formation $\tau$. It is important to notice, that the value of $\tau$ can be obtained, though indirectly, from experiments [7, 54]. Within the proposed model, confinement of cluster growth due to complex formation is taken into account by the following modification:

$$
w_{n, m}^{( \pm)}(t)=w_{n, m}^{( \pm)}(t) e^{-\frac{t}{\tau}}
$$

According to Eq. (2.21) the probability of cluster growth is proportional to the concentration of single particles in solution, $f(1, t)$. This concentration monotonously decreases with time-fullerenes from solution transfer to clusters, thus, the probabilities of growth also decrease with time. Asymptotically the probabilities tend not to zero, but to a certain constant, which corresponds to the detailed balance in the system, for example for (2.21) we have $w_{n-1, n}^{(+)} \rightarrow 4 \pi D c_{\mathrm{eq}} r n^{1 / 3}$. On the opposite, the new transition probabilities (introduced via (3.3)), tend to zero after long period of time $t \gg \tau$. Let us note, that similar models were already developed, for example, in $[55,56]$ during investigations of kinetics of cluster growth at the Ostwald ripening stage in porous materials under elastic stresses.

In polar fullerene solutions $\left(\mathrm{C}_{60} / \mathrm{NMP}, \mathrm{C}_{60} /\right.$ pyridine) different researchers reported the growth of huge clusters, with sizes from $100 \mathrm{~nm}$, up to $500 \mathrm{~nm}$ 

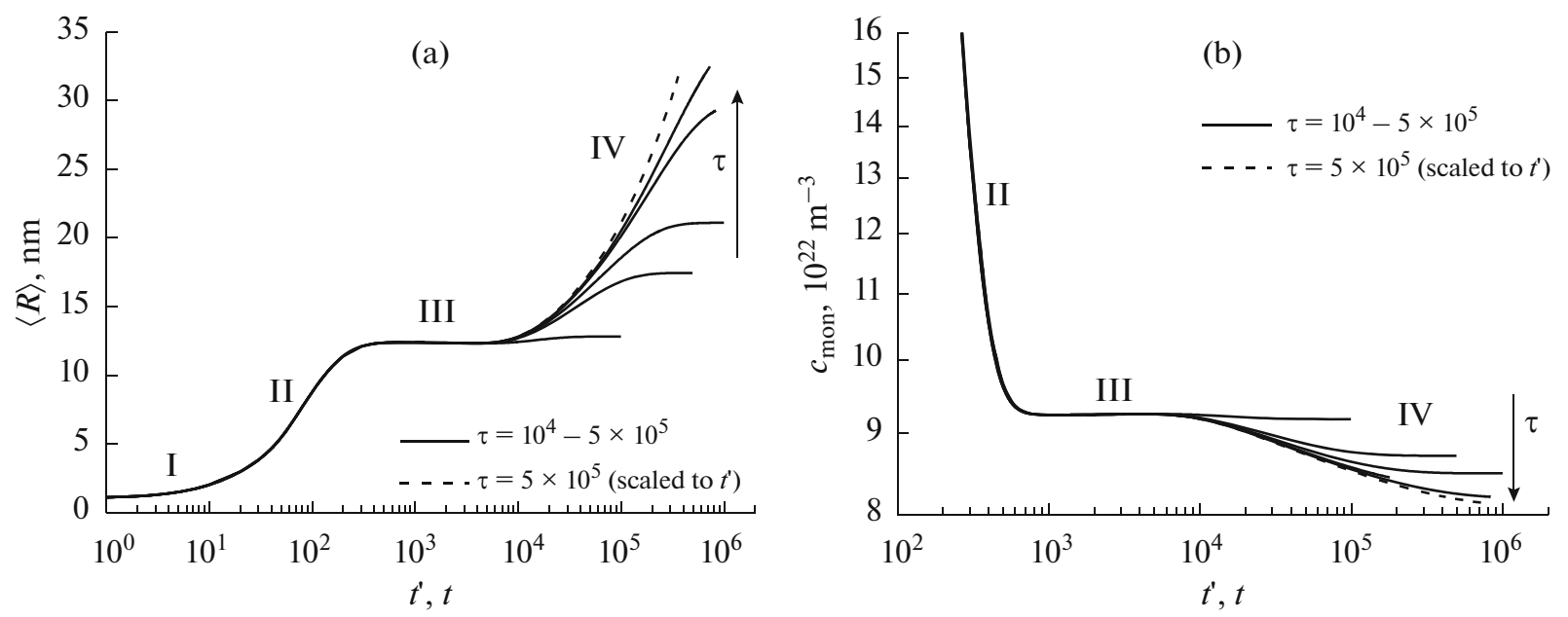

Fig. 6. The results of numerical calculations of kinetics of cluster growth in a model solution for different values of $\tau$. Dashed curves show the dependencies for the reduced time scale (see (3.4)). (a) Time evolution of the mean cluster radius in solution, (b) Time evolution of the monomers concentration.

$[48,49]$. An estimate of the number of particles in the cluster gives: $n \sim 10^{6}$ for $100 \mathrm{~nm}, n \sim 4 \times 10^{6}$ for $150 \mathrm{~nm}$, and $n \sim 10^{7}$ for $200 \mathrm{~nm}$. Numerical calculations of the full dependence $f(n, t)$, thus, require solution for times $t \gg \tau$ of the system of at least $10^{7}$ kinetic equations, an overly time-consuming task even for the modern computational resources. In [7, 42, 52] a method of calculations of $f(n, t)$ for the considered here model with complex formation was developed. It was shown, that if the right scaling of the time axis is made, the average size of the particles reduces to the Slezov's " $t^{1 / 3}$ law" [32].

From experimental data, the value of model parameter can be estimated: $\tau \sim 10^{8}-10^{9}$ [42] (reduced time-scale is used). For the full evaluation of $f(n, t)$ function for these values of $\tau$, first we have consequently investigated a set of model systems with smaller times of complex formation (the set of $\tau$ values: $\left.10^{4}, 5 \times 10^{4}, 10^{5}, 3 \times 10^{5}, 5 \times 10^{5}\right)$. By analyzing the dependencies of average radius of clusters $\langle R\rangle$ and monomer concentration, $f(1, t)$, for different $\tau$ it was shown that the scaling of time using the expression:

$$
t^{\prime}=\tau\left(1-e^{-t / \tau}\right)
$$

reduces all the curves to a single master-curve (Fig. 6). For large values of time $t^{\prime}$ the dependence (2.26) holds. In addition, in [42] it was shown that cluster size distribution functions at late stages of growth (stage IV), are identical to the functions (2.27), analytically obtained by Slezov. The time in expressions (2.26), (2.27) must be taken with account of reducing Eq. (3.4). Thus, to obtain the full evolution of the cluster size distribution function for any, no matter how large, value of $\tau$, we take the numerical calculation results for stages of growth I-III, and the transition from stage III to IV and supplement the results with analytical functions for later stages.

Using the method proposed in [42], calculations of the whole evolution of the distribution function $f(R, t)$ for a model system, corresponding to solution of $C_{60}$ in $\mathrm{N}$-methylpyrrolidone were made. The results of calculations are shown on Fig. 7. The obtained distribution functions qualitatively correspond to polar fullerene $\mathrm{C}_{60}$ solutions [48, 50, 57]. Yet, it should also be noted, that in the late structural investigations of clusters in these solutions [51] their complicated (several levels) structure was revealed. Thus, further modifications of theoretical models are probably required.

\subsection{A Model of Clusters Decomposition on Dissolution by Water}

Lately, the results obtained in Section 3.2 were applied for description of an interesting effect of clusters decomposition after dissolution of fullerene $\mathrm{C}_{60}$ polar solution by water [41]. This effect was discovered in 2004 via neutron scattering $[58,59]$, it has a critical character-decomposition occurs only after the amount of water added exceeds $40 \mathrm{vol} \%$. Decomposition takes place right after dissolution. Using numerical calculations [60] it was shown, that in case of linear dependencies of systems properties on the amount of added water no critical effect exists. Thus in further research [41] the non-linear dependencies were considered.

On dissolving a colloidal solution, we change its physical and chemical properties. This also influences the cluster size distribution function. First, define the 

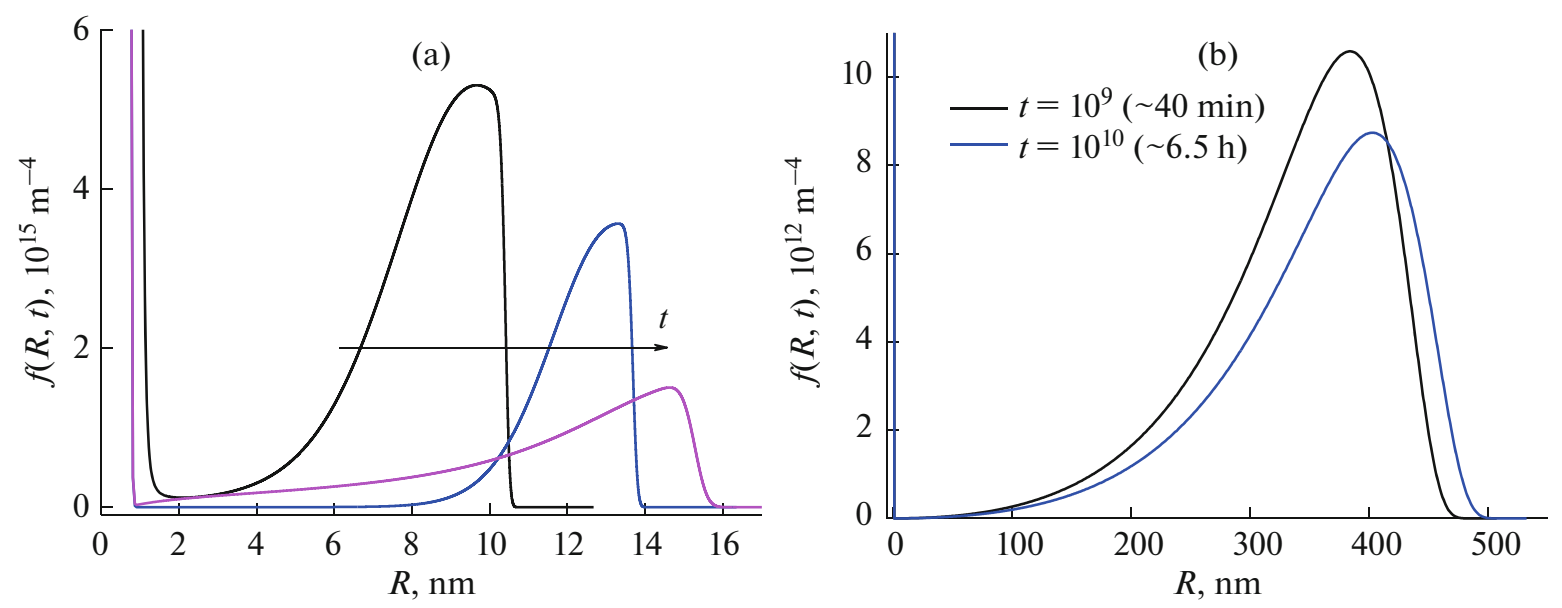

Fig. 7. Cluster size distribution function for $\mathrm{C}_{60}$ in model polar solution. (a) Initial stages of growth (for times less than one second); (b) Growth of huge clusters, time of evolution denoted on the graph.

model parameter $X$, that describes the amount of water added:

$$
X=\frac{V_{\mathrm{H}_{2} \mathrm{O}}}{V_{\mathrm{H}_{2} \mathrm{O}}+V_{\mathrm{SOL}}}
$$

where $V_{\mathrm{H}_{2} \mathrm{O}}$ is the volume of water added, $V_{\mathrm{SOL}}$ is the volume of solution prior to dissolution. By definition the values of $X$ lie on a segment [0,1], where 0 and 1 are the limiting values: $X=0$ corresponds to the case when solution was not dissolved; $X=1$ - the case of an infinite amount of added water. On dissolution, the properties of the model system change as follows:

(1) The cluster size distribution function $f(n, t)$ must be multiplied by a factor, reflecting the increase of solvent volume:

$$
f^{\prime}(n, t)=f(n, t) \frac{1-X}{1+X} .
$$

(2) The values of parameters $D, c_{\mathrm{eq}}, \alpha_{2} / k_{\mathrm{B}} T$ and $\tau$ change:

(2.1) The diffusion coefficients of clusters in water and initial solvent can be estimated using Stokes-Einstein equation, the difference is less than two times. In the model approach, we will consider that $D$ changes linearly:

$$
D^{\prime}=D_{\mathrm{SOL}}+X\left(D_{\mathrm{H}_{2} \mathrm{O}}-D_{\mathrm{SOL}}\right),
$$

where $D^{\prime}$ is the value of diffusivity in the mixed solvent.

(2.2) The change of $\tau$ was not considered in the model [41]. However, after dissolution we recover the initial values of probabilities $w^{+}$and $w^{-}$(reset to zero the exponent in (3.3)).

(2.3) The value of $\alpha_{2}$ influences the critical cluster size in the system. As a first approximation, we can consider that the ratio of $\alpha_{2}$ in initial solvent and water is equal to the ratio of dielectric permittivities of these liquids. In a mixture, the value of surface term $\alpha_{2}$ was also considered to change linearly with $X$.

(2.4) The most significant part for describing the clusters decomposition on dissolution is in the correct modeling of $c_{\text {eq }}(X)$ change. Fullerene is a hydrophobic molecule, yet after dissolution of its polar solution, the fullerene complexes with solvent molecules, having a non-zero solubility in $\mathrm{H}_{2} \mathrm{O}$, may transfer to water. We suppose that the concentration $c_{\text {eq }}$ continuously decreases from $c_{\mathrm{eq}}^{\mathrm{SOL}}$ at $X=0$ to a certain, relatively small, unknown value $c_{\mathrm{eq}}^{\mathrm{H}_{2} \mathrm{O}}$ for $X=1$. The choice of the dependence is based on the results of investigations of application of $\mathrm{N}$-methylpyrrolidone solvent for improvement of drugs solubility in water [61, 62]. The expression for concentrations corresponding to this behavior of NMP has the following form:

$$
\frac{c_{\mathrm{eq}}^{\mathrm{MIX}}}{c_{\mathrm{eq}}^{\mathrm{SOL}}}=\left(\frac{c_{\mathrm{eq}}^{\mathrm{H}_{2} \mathrm{O}}}{c_{\mathrm{eq}}^{\mathrm{SOL}}}\right)^{X} \text {. }
$$

The concentration $c_{\mathrm{eq}}^{\mathrm{H}_{2} \mathrm{O}}<c_{\mathrm{eq}}^{\mathrm{SOL}}$, thus, is the new model parameter. For further estimates, we define a non-dimensional model parameter $S=c_{\mathrm{eq}}^{\mathrm{SOL}} / c_{\mathrm{eq}}^{\mathrm{H}_{2} \mathrm{O}}$.

The modeling of dissolution was performed using the cluster size distribution functions obtained in Section 3.2. A model system with a relatively small value $\tau=10^{5}$ was considered (the results of modeling may be extrapolated to arbitrary large values of $\tau$ ). Let us also reset time to $t=0$ after dissolution for convenience. The cluster reorganization occurs right after dissolution, thus we are interested in periods of time with $t \ll \tau$.

The calculated evolution of the average cluster radius in the binary $\mathrm{SOL}-\mathrm{H}_{2} \mathrm{O}$ mixture for different $X$ values on the whole segment $[0,1]$ is presented on Fig. 8. On weak dissolutions by water the cluster state 


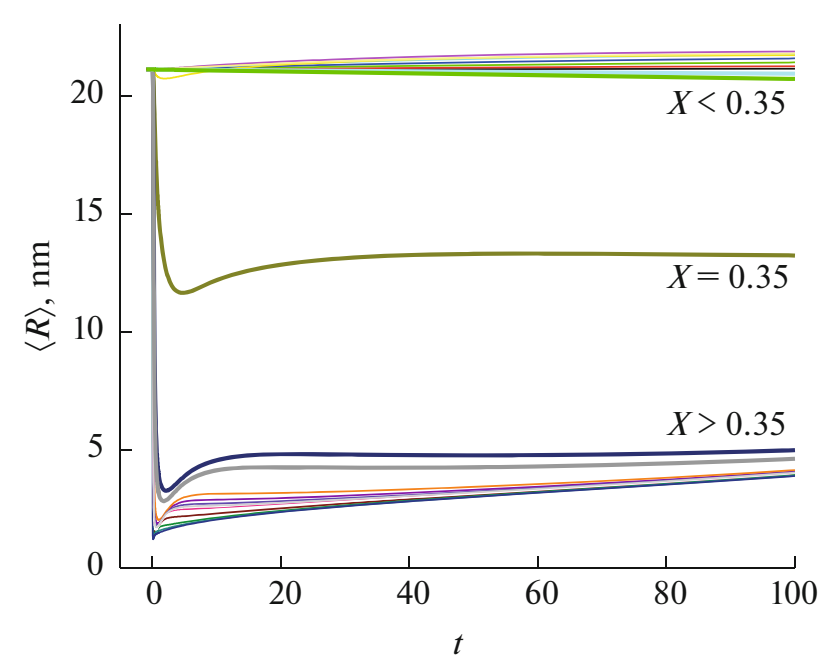

Fig. 8. A model of dissolution of solution of segregating particles with water: time dependence of the average clusters radius after dissolution for different values of $X$ [41].

does not change. Yet, starting with a certain critical $X$ value, a rapid decrease of cluster sizes on water dissolution occurs (the region around $X_{\mathrm{c}}=0.35$ on Fig. 8). Let us note that for $X>0.4$ the average cluster size rapidly decreases to a certain value which further does not depend on $X$. Further growth of average cluster size is a slow process, mostly determined by $D$ ' value.

The critical value $X_{\mathrm{c}}$, that determines the decomposition effect, depends strongly on parameter $S$ (ratio of $c_{\mathrm{eq}}^{\mathrm{H}_{2} \mathrm{O}}$ and $c_{\mathrm{eq}}^{\mathrm{SOL}}$ concentrations). The numerical calculations were performed for different $S$ values. The dependence of critical value of dissolution coefficient, $X_{\mathrm{c}}$, on concentrations ratio is presented on Fig. 9 [41].

The reason for cluster decomposition is the dissolution driven change of ratio between saturation concentration $\left(c_{\mathrm{eq}}^{\mathrm{MIX}}\right)$ in binary solvent to the monomers concentration, $f^{\prime}(n=1)$. In a certain range of $X$ values a strong supersaturation may be realized $\left(f^{\prime}(n=1) \gg c_{\mathrm{eq}}^{\mathrm{MIX}}\right)$. In this $X$ range the nucleation and growth of clusters occurs once again (early stages), which leads to formation of a new peak in $f^{\prime}(n, t)-\mathrm{a}$ bimodal distribution is formed (Fig. 10), with both populations of clusters growing slowly in size. This leads to a decrease of the registered average size of clusters in solution. In fact, some monomers are detached from large clusters and start forming new aggregates. These results of the model qualitatively correspond to experimental observations showing that the decomposition of huge clusters proceeds by detachment of separate $\mathrm{C}_{60}$ molecules.

Thus, the proposed model describes the critical effect of cluster decomposition on dissolution of col-

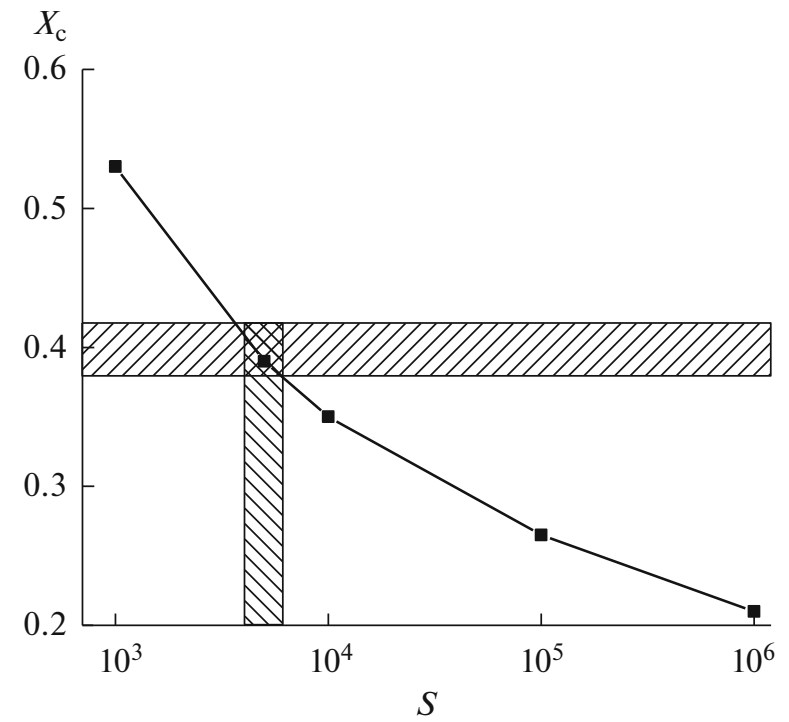

Fig. 9. Dependence of the critical value of dissolution parameter $X_{\mathrm{c}}$ on model parameter $S$. The hatched areas highlight the parameter values, corresponding to experimental data.

loidal solution by water [41]. The results are obtained employing the hypothesis on power-law dependence (3.7) of the saturation concentration of dissolved particles in the binary solvent. Let us note, that the modeled effect has a general character and can be observed in the systems, where Ostwald's ripening stage takes a sufficiently long time. Addition to the system of a solvent, in which particles behave in a lyophobic manner, may considerably reduce $c_{\text {eq }}$. This will lead to the development of the bimodal cluster size distributions.

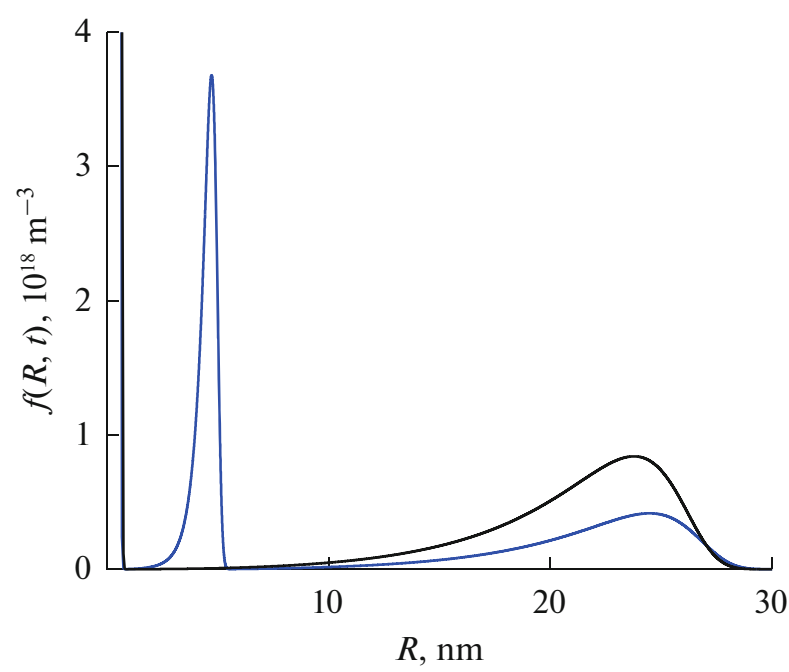

Fig. 10. Cluster size distribution function in solution prior (black curve) and after (blue curve) dissolution by water in the range of the critical decomposition effect [41]. 


\section{CONCLUSIONS}

In the present work, the results of application of theory of clusters formation and growth for description of kinetic processes in fullerene solutions were presented. Even for a qualitative description of the observed effects, some modifications of the theory reviewed in Section 2 were required. In conclusion, we discuss several questions connected with perspectives of applications of this method to fullerene solutions and nanoparticles aggregation in liquid media in general. Let us note that applications of nucleation theory for description of aggregation in solutions of proteins and other organic molecules or salts are actively discussed [63]. The classical approach is compared with the recently proposed two-steps nucleation mechanism $[63,64]$. A similar approach was also discussed in one of our works on investigation of polar fullerene solutions [65].

A separate notice regarding applications of computer simulations for investigation of nucleation and growth processes should be made. The molecular dynamics method is actively used at present for studying the irreversible processes in condensed matter physics [66], particularly the nucleation phenomena. A review of actual results for nucleation in solutions is given in [67]. It is denoted, that molecular dynamics is an effective method that allows detailed investigation of initial stages of nuclei formation and growth in model systems at the microscopic level, also allowing to compute, for example, the nucleation rate and to probe the applicability of classical and modified theories in respect to simulations. A number of questions connected with method applications is discussed, particularly the necessity of accurate choice and further elaboration of interaction potentials, and sampling methods. Examples of investigations of crystallization and melting of particles interacting by Lennard-Jones potential can be found in $[68,69]$. It should be noted however, that investigations of growth of large clusters on long timescales are limited by the computational power of the modern resources. Consequently, a detailed microscopic investigation of growth of huge $\mathrm{C}_{60}$ aggregates is probably not possible. Thus, the kinetic equations, discussed in this review, play and important and indispensable role. On the other hand, the molecular dynamics method finds its application in investigation of fullerene solutions in a following way. Already in [70] an investigation of stability of small clusters of $\mathrm{C}_{60}$, interacting via a classical (for fullerenes) Girifalco interaction potential is reported. The modern works are mostly concentrated on investigations of structure and dynamic characteristics of solvate shells formed around single fullerene molecules in solution. For example, for carbon disulfide these investigations were made in [39, 46], for other three low-polar solvents-in [71], and for ethanol-in [72]. These results provide important information, which can be further accounted for in analysis of experimental data and modeling of fullerenes aggregation.

Returning to applications of nucleation and growth approach, modifications of the expression for the work of cluster formation in solution, $\Delta G(n)$, may be considered in order to move towards a quantitative theory. Presently, the size of the critical cluster in $\mathrm{C}_{60}$ solutions is $\sim 15-20$ monomers, thus the expressions for $\Delta G(n)$ for small $n$ values may play a role. Here, for example, application of atomistic approaches may be considered. Also, the expressions for the driving force in the considered systems can be discussed. Application of the expression holding for ideal solution (2.20) introduces an additional parameter $c_{\text {eq }}$, which is different from the saturation concentration in fullerene solutions [7]. For numerical calculations we suppose that $c_{\text {eq }} \ll c_{\text {sat }}$, to account for the fact that at different conditions aggregation is observed even for the dissolved (unsaturated) solutions. The question on maximum value of $c_{\text {eq }}$ was discussed in [50]. Presently there is still no reliable way to estimate the value of this parameter. Consideration of different expressions for $\Delta \mu$, thus, is justified.

Another question is connected with expansion of Eqs. (2.8) for the account of aggregation of clusters with each other. That is a modification of the classical approach, proposed by the nucleation theory. Indeed, at the early stages (nucleation, start of clusters growth), the prevailing processes are the attachment/detachment of monomers. Yet, as the number of aggregates increases in the system, their interactions must be taken into account. The cluster-cluster collision probabilities, negligibly small at the nucleation stage, continuously grow, and at stage IV (Fig. 4) may be important. The form of equations accounting for this type of processes is the following:

$$
\begin{aligned}
\frac{d f(n, t)}{d t}= & \frac{1}{2} \sum_{m=1}^{n-1} \alpha_{m, n-m} \beta_{m, n-m} f(m, t) f(n-m, t) \\
& -\sum_{m=1}^{n_{\max }} \alpha_{n m} \beta_{n m} f(n, t) f(m, t) \\
- & S_{n} f(n, t)+\sum_{m=n+1}^{n_{\max }} \Gamma_{m n} S_{m} f(m, t),
\end{aligned}
$$

where $\alpha_{n m}$ and $\beta_{n m}$ are effectivity and frequency of collisions between particles (defined by $n$ and $m$ ), $S_{n}$ is the decay rate, $\Gamma_{m n}$ is the distribution function of decay fragments. Effectivity of collisions $\alpha$ defines the part of collisions leading to aggregation. The expressions for most of the parameters may be obtained from the classical nucleation theory.

Investigations of this system of equations are actual for the description of fullerene solutions also in connection with the results of structural investigations of $\mathrm{C}_{60}$ aggregates in solutions, which show that clusters have a two-level structure [51]. The compact small sized quasispherical fullerene clusters form loose frac- 
tal aggregates with sizes of hundreds of nanometers. In this case, Eqs. (2.8) can be used for description of first stage of systems evolution-formation of compact aggregates, and the later equations-for description of growth of fractal clusters.

Finally, the recent experimental results on growth of clusters in fullerene solutions in toluene should be noted [73]. In this work, generalizing results of previous research, it is shown, that aggregation in low-polar fullerene solutions occurs as a consequence of formation of $\mathrm{C}_{60} \mathrm{O}_{x}$ oxides. These covalent complexes are the reason of clusters growth. Our investigations with other two solvents-benzene and chlorobenzene allow suggesting that this phenomenon is general for fullerene molecules. From the theoretical viewpoint, one must consider then a model system, containing two types of segregating particles of similar size-type A (plain fullerene $\mathrm{C}_{60}$ ) and type $\mathrm{B}$ (the $\mathrm{C}_{60} \mathrm{O}$ oxide). The attraction between particles $\mathrm{A}$ is not sufficient for their aggregation, while particles $\mathrm{B}$ can form aggregates with each other, as well as with particles of type A. Development of this model for description of kinetics of cluster growth in these systems requires modification of the considered here equations.

It can be summarized, that kinetic processes in fullerene solutions present an interesting system for description via the system of kinetic equations for cluster formation and growth.

\section{REFERENCES}

1. H. W. Kroto, J. R. Heath, S. C. O'Brien, R. F. Curl, and R. E. Smalley, " $\mathrm{C}_{60}$ : Buckminsterfullerene," Nature 318, 162 (1985).

2. A. V. Eletskii and B. M. Smirnov, "Fullerenes," Phys. Usp. 36 (3), 202-224 (1993).

3. R. S. Ruoff, D. S. Tse, R. Malhotra, and D. C. Lorents, "Solubility of $\mathrm{C}_{60}$ in a variety of solvents," J. Phys. Chem. 97, 3379-3383 (1993).

4. M. V. Avdeev, V. L. Aksenov, and T. V. Tropin, "Models of cluster formation in solution of fullerenes," Russ. J. Phys. Chem. A 84, 1273-1283 (2010).

5. N. O. Mchedlov-Petrossyan, N. N. Kamneva, Y. T. M. Al-Shuuchi, A. I. Marynin, and O. S. Zozulia, "Formation and ageing of the fullerene $\mathrm{C}_{60}$ colloids in polar organic solvents," J. Mol. Liq. 235, 98-103 (2017).

6. N. O. Mchedlov-Petrossyan, "Fullerenes in liquid media: An unsettling intrusion into the solution chemistry," Chem. Rev. 113, 5149-5193 (2013).

7. T. V. Tropin, M. V. Avdeev, N. Jargalan, M. O. Kuzmenko, and V. L. Aksenov, "Kinetics of cluster growth in fullerene solutions of different polarity," Mod. Probl. Phys. Liquid Syst. 36, 249-272 (2019).

8. M. Volmer, Kinetik der Phasenbildung (Steinkopff, Dresden, 1939).

9. J. W. Gibbs, "On the equilibrium of heterogeneous substances," Trans. Connect. Acad. Arts Sci. 3, 108-248 (1876).
10. J. W. Gibbs, "On the equilibrium of heterogeneous substances," Trans. Connect. Acad. Arts Sci. 3, 343-524 (1878).

11. M. Volmer and A. Weber, "Keimbildung in übersättigten Gebilden,” Z. Phys. Chem. 119U (1) (1926).

12. L. Farkas, "Keimbildungsgeschwindigkeit in übersättigten Dämpfen,” Z. Phys. Chem. 125U (1) (1927).

13. R. Kaischew and I. N. Stranski, "Zur kinetischen Ableitung der Keimbildungsgeschwindigkeit," Z. Phys. Chem. 26 (1), 317-326 (1934).

14. R. Kaischew and I. N. Stranski, "Zur Theorie der linearen Kristallisationsgeschwindigkeit," Z. Phys. Chem. 170 (1), 295-299 (1934).

15. R. Becker and W. Döring, "Kinetische Behandlung der Keimbildung in übersättigten Dämpfen," Ann. Phys. (New York) 416, 719-752 (1935).

16. Ya. B. Zeldovich, "On the theory of new phase formation. Cavitation," Zh. Eksp. Teor. Fiz. 11 (11-12), 525-538 (1942).

17. Ya. I. Frenkel, Kinetic Theory of Liquids (New York, 1955).

18. D. Turnbull and J. C. Fisher, "Rate of nucleation in condensed systems,” J. Chem. Phys. 17, 71-73 (1949).

19. I. S. Gutzow and J. W. P. Schmelzer, The Vitreous State: Thermodynamics, Structure, Rheology, and Crystallization, 2nd ed. (Springer Berlin Heidelberg, 2013).

20. Nucleation Theory and Applications, Ed. by J. W. P. Schmelzer (Wiley-VCH, 2005).

21. L. D. Landau and E. M. Lifshitz, Statistical Physics, 3rd ed. (Pergamon Press, Oxford, 1980).

22. J. W. P. Schmelzer and A. S. Abyzov, "Crystallization of glass-forming liquids: Thermodynamic driving force," J. Non. Cryst. Solids 449, 41-49 (2016).

23. J. W. P. Schmelzer, G. Ropke, and V. B. Priezzhev, Nucleation Theory and Applications, Ed. by J. W. P. Schmelzer, G. Ropke, and V. B. Priezzhev (JINR, Publ., Dubna, 1999).

24. V. V. Slezov and J. W. P. Schmelzer, "Kinetics of formation of a phase with an arbitrary stoichiometric composition in a multicomponent solid solution," Phys. Rev. E 65 (3), 1-13 (2002).

25. V. V. Slezov and J. W. P. Schmelzer, "Kinetics of formation and growth of a new phase with a definite stoichiometric composition,” J. Phys. Chem. Solids 55, 243251 (1994).

26. V. V. Slezov, Y. J. Tkatch, and J. W. P., Schmelzer, "The kinetics of decomposition of solid solutions," J. Mater. Sci. 32, 3739-3747 (1997).

27. J. Möller, K. I. Jacob, and J. W. P. Schmelzer, "Ostwald ripening in porous viscoelastic materials," J. Phys. Chem. Solids 59, 1097-1103 (1998).

28. D. T. Wu, "Nucleation theory," in Solid State Physics (New York, NY, 1996), pp. 37-187.

29. V. V. Slezov, Kinetics of First-Order Phase Transitions (Wiley-VCH, Weinheim, 2009).

30. V. L. Aksenov, T. V. Tropin, M. V. Avdeev, V. B. Priezzhev, and J. W. P. Schmelzer, "Kinetics of cluster growth in fullerene molecular solutions," Phys. Part. Nucl. 36, S52-S61 (2005).

31. J. W. P. Schmelzer and J. Möller, "Evolution of the cluster size-distribution function for Ostwald ripening 
in viscoelastic media," Phase Transitions 38, 261-272 (1992).

32. V. V. Slezov and V. V. Sagalovich, "Diffusive decomposition of solid solutions," Sov. Phys. Usp. 30, 23-45 (1987).

33. I. M. Lifshitz and V. V. Slezov, "Kinetics of diffusive decomposition of supersaturated solid solutions," Sov. Phys. JETP 35, 331-339 (1959).

34. M. V. Korobov and A. L. Smith, "Solubility of fullerenes," in Fullerenes: Chemistry, Physics and Technology, Ed. by K. M. Kadish and R. S. Ruoff (John Wiley \& Sons, 2000), pp. 53-89.

35. K. N. Semenov, N. A. Charykov, V. A. Keskinov, A. K. Piartman, A. A. Blokhin, and A. A. Kopyrin, "Solubility of light fullerenes in organic solvents," J. Chem. Eng. Data 55, 13-36 (2010).

36. V. N. Bezmel'nitsyn, A. V. Eletskii, and M. V. Okun', "Fullerenes in solutions," Phys.-Usp. 168, 1195 (1998).

37. K. A. Affholter, S. J. Henderson, G. D. Wignall, G. J. Bunick, R. E. Haufler, and R. N. Compton, "Structural characterization of $\mathrm{C}_{60}$ and $\mathrm{C}_{70}$ fullerenes by small-angle neutron scattering," J. Chem. Phys. 99, 9224 (1993).

38. N. O. Mchedlov-Petrossyan, "Fullerenes in molecular liquids. Solutions in "good" solvents: Another view," J. Mol. Liq. 161, 1-12 (2011).

39. M. V. Avdeev, T. V. Tropin, I. A. Bodnarchuk, S. P. Yaradaikin, L. Rosta, V. L. Aksenov, and L. A. Bulavin, "On structural features of fullerene $\mathrm{C}_{60}$ dissolved in carbon disulfide: Complementary study by small-angle neutron scattering and molecular dynamic simulations," J. Chem. Phys. 132, 164515 (2010).

40. T. V. Tropin, V. B. Priezzhev, M. V. Avdeev, J. W. P. Schmelzer, and V. L. Aksenov, "Fullerene cluster formation in carbon disulfide and toluene," Fullerenes, Nanotubes, Carbon Nanostruct. 14, 481488 (2006).

41. T. V. Tropin and V. L. Aksenov, "Theoretical study of the effect of decrease of cluster size on dilution of a solution with water," JETP 128, 274-280 (2019).

42. T. V. Tropin, M. V. Avdeev, and V. L. Aksenov, "Modeling of the evolution of cluster-size distribution functions in polar fullerene $\mathrm{C}_{60}$ solutions," J. Surf. Invest.: X-ray Synchrotron Neutron Tech. 13, 82-86 (2019).

43. T. Tomiyama, S. Uchiyama, and H. Shinohara, "Solubility and partial specific volumes of $\mathrm{C}_{60}$ and $\mathrm{C}_{70}$," Chem. Phys. Lett. 264, 143-148 (1997).

44. T. V. Tropin, M. V. Avdeev, and V. L. Aksenov, "Smallangle neutron scattering study of $\mathrm{C}_{60} / \mathrm{CS}_{2}$ solutions," Fullerenes, Nanotubes, Carbon Nanostruct. 16, 616621 (2008).

45. T. V. Tropin, M. V. Avdeev, and V. L. Aksenov, "Smallangle neutron scattering data on $\mathrm{C}_{60}$ clusters in weakly polar solutions," Cryst. Rep 52, 483-486 (2007).

46. M. V. Avdeev, T. V. Tropin, V. L. Aksenov, L. Rosta, and M. T. Kholmurodov, "Formation of fullerene clusters in carbon disulfide: Data on small-angle neutron scattering and molecular dynamics," J. Surf. Invest.: X-ray Synchrotron Neutron Tech. 2, 819-825 (2008).

47. T. V. Tropin, M. V. Avdeev, V. B. Priezzhev, and V. L. Aksenov, "Nonmonotonic behavior of the con- centration in the kinetics of dissolution of fullerenes," JETP Lett. 83, 399-404 (2006).

48. N. P. Yevlampieva, Yu. F. Biryulin, E. Yu. Melenevskaja, V. N. Zgonnik, and E. I. Rjumtsev, "Aggregation of fullerene $\mathrm{C}_{60}$ in N-methylpyrrolidone," Colloids Surf. A Physicochem. Eng. Asp. 209, 167-171 (2002).

49. O. A. Kyzyma, L. A. Bulavin, V. L. Aksenov, T. V. Tropin, M. V. Avdeev, M. V. Korobov, S. V. Snegir, and L. Rosta, "Aggregation in $\mathrm{C}_{60} / \mathrm{NMP}, \mathrm{C}_{60} / \mathrm{NMP} /$ Water and $\mathrm{C}_{60} / \mathrm{NMP} /$ toluene mixtures," Fullerenes, Nanotubes, Carbon Nanostruct. 16, 610-615 (2008).

50. O. A. Kyzyma, M. V. Korobov, M. V. Avdeev, V. M. Garamus, S. V. Snegir, V. I. Petrenko, V. L. Aksenov, and L. A. Bulavin, "Aggregate development in $\mathrm{C}_{60} / \mathrm{N}$-methyl-2-pyrrolidone solution and its mixture with water as revealed by extraction and mass spectroscopy," Chem. Phys. Lett. 493, 103-106 (2010).

51. S. V. Snegir, T. V. Tropni, O. A. Kyzyma, M. O. Kuzmenko, V. I. Petrenko, V. M. Garamus, M. V. Korobov, M. V. Avdeev, and L. A. Bulavin, "On a specific state of $\mathrm{C}_{60}$ fullerene in $\mathrm{N}$-methyl-2-pyrrolidone solution: Mass spectrometric study,” Appl. Surf. Sci. 481, 15661572 (2019).

52. T. V. Tropin, M. V. Avdeev, O. A. Kyzyma, R. A. Yeremin, N. Jargalan, M. V. Korobov, and V. L. Aksenov, "Towards description of kinetics of dissolution and cluster growth in $\mathrm{C}_{60}$ /NMP solutions," Phys. Status Solidi B 248, 2728-2731 (2011).

53. T. V. Tropin, N. Jargalan, M. V. Avdeev, O. A. Kyzyma, R. A. Eremin, D. Sangaa, and V. L. Aksenov, "Kinetics of cluster growth in polar solutions of fullerene: Experimental and theoretical study of $\mathrm{C}_{60} / \mathrm{NMP}$ solution," J. Mol. Liq. 175, 4-11 (2012).

54. N. Jargalan, T. V. Tropin, M. V. Avdeev, and V. L. Aksenov, "Investigation and modeling of evolution of $\mathrm{C}_{60}$ /NMP solution UV-Vis spectra," Nanosyst. Phys. Chem. Math. 7, 99-103 (2016).

55. J. W. P. Schmelzer and I. S. Gutzow, "On the kinetic description of Ostwald ripening in elastic media," Z. Phys. Chem. 269, 753-767 (1988).

56. R. Pascova, I. S. Gutzow, and J. W. P. Schmelzer, "A model investigation of the process of phase formation in photochromic glasses,” J. Mater. Sci. 25, 921931 (1990).

57. A. Mrzel, A. Mertelj, A. Omerzu, M. Copic, and D. Mihailovic, "Investigation of encapsulation and solvatochromism of fullerenes in binary solvent mixtures," J. Phys. Chem. B 103, 11256-11260 (1999).

58. V. L. Aksenov, M. V. Avdeev, T. V. Tropin, M. V. Korobov, N. V. Kozhemyakina, N. V. Avramenko, and L. Rosta, "Formation of fullerene clusters in the system $\mathrm{C}_{60} / \mathrm{NMP} /$ water by SANS," Phys. B Condens. Matter 385-386, 795-797 (2006).

59. T. V. Tropin, T. O. Kyrey, O. A. Kyzyma, A. V. Feoktistov, M. V. Avdeev, L. A. Bulavin, L. Rosta, and V. L. Aksenov, "Experimental investigation of $\mathrm{C}_{60} / \mathrm{NMP} /$ toluene solutions by UV-Vis spectroscopy and small-angle neutron scattering," J. Surf. Invest.: X-ray Synchrotron Neutron Tech. 7, 1-4 (2013).

60. V. L. Aksenov, T. V. Tropin, O. A. Kyzyma, M. V. Avdeev, M. V. Korobov, and L. Rosta, "Forma- 
tion of $\mathrm{C}_{60}$ fullerene clusters in nitrogen-containing solvents," Phys. Solid State 52, 1059-1062 (2010).

61. P. Jain and S. H. Yalkowsky, "Solubilization of poorly soluble compounds using 2-pyrrolidone," Int. J. Pharm. 342, 1-5 (2007).

62. R. Sanghvi, R. Narazaki, S. G. Machatha, and S. H. Yalkowsky, "Solubility improvement of drugs using N-methyl pyrrolidone," Am. Assoc. Pharm. Sci. 9, 366-376 (2008).

63. R. G. Davey, S. L. M. Schroeder, and J. H. ter Horst, "Nucleation of Organic Crystals-A Molecular Perspective," Angew. Chem. Int. 52, 2-16 (2013). https://doi.org/10.1002/anie.201204824

64. R. J. Davey, S. L. M. Schroeder, and J. H. Ter Horst, "Nucleation of organic crystals - A molecular perspective," Angew. Chem. Int. Ed. 52, 2167-2179 (2013).

65. T. V. Tropin, M. V. Avdeev, O. A. Kyzyma, and V. L. Aksenov, "Nucleation theory models for describing kinetics of cluster growth in $\mathrm{C}_{60}$ /NMP solutions," Phys. Status Solidi 247, 3022-3025 (2010).

66. G. E. Norman and V. V. Stegailov, "Stochastic theory of the classical molecular dynamics method," Math. Mod. Comp. Simul. 5, 305-333 (2013).

67. G. C. Sosso, J. Chen, S. J. Cox, M. Fitzner, P. Pedevilla, A. Zen, and A. Michaelides, "Crystal nucleation in liquids: Open questions and future challenges in molec- ular dynamics simulations," Chem. Rev. 116, 70787116 (2016).

68. A. O. Tipeev, E. D. Zanotto, and J. P. Rino, "Diffusivity, interfacial free energy, and crystal nucleation in a supercooled Lennard-Jones liquid," J. Phys. Chem. C 122, 28884-28894 (2018).

69. V. G. Baidakov and A. O. Tipeev, "Molecular dynamics simulation of homogeneous nucleation in a superheated Lennard-Jones crystal,” J. Non-Cryst. Solids 503504, 302-307 (2019).

70. C. Rey, L. J. Gallego, and J. A. Alonso, "Moleculardynamics study of the structure, binding energy, and melting of small clusters of fullerene molecules using Girifalco's spherical model," Phys. Rev. B 49, 84918494 (1994).

71. C. I. Wang, C. C. Hua, and S. A. Chen, "Dynamic solvation shell and solubility of $\mathrm{C}_{60}$ in organic solvents," J. Phys. Chem. B 118, 9964-9973 (2014).

72. T. Malaspina, E. E. Fileti, and R. Rivelino, "Structure and UV-Vis spectrum of $\mathrm{C}_{60}$ fullerene in ethanol: A sequential molecular dynamics/quantum mechanics study," J. Phys. Chem. B 111, 11935-11939 (2007).

73. R. Dattani, K. F. Gibson, S. Few, A. J. Borg, P. A. DiMaggio, J. Nelson, S. G. Kazarian, and J. T. Cabral, "Fullerene oxidation and clustering in solution induced by light," J. Colloid Interface Sci. 446, 24-30 (2015). 